\title{
OPTIONS FOR THE NEUTRON LIFETIME MEASUREMENTS IN TRAPS
}

\author{
Yu. N. Pokotilovski
}

Joint Institute for Nuclear Research, Dubna, Russia

\begin{abstract}
Different geometries for the neutron lifetime measurements by the method of ultracold neutron storage in material traps and additional possibilities for the neutron storage in the magnetic storage ring are considered.

PACS: 07.05.Fb, 13.30.-a, 13.30.Ce, 21.10.Tg

\section{INTRODUCTION}

The neutron lifetime is still one of the least accurately measured fundamental nuclear constants. It is known that precision measurement of the neutron lifetime along with other neutron decay experiments has significant impact on the Standard Model parameters. Recent reviews are published in [1-3].

There are two principal ways to measure the neutron lifetime. In a neutron beam experiment the $\beta$-activity of a beam of cold neutrons is measured, and the neutron lifetime precision is determined by the accuracy of absolute measurement of neutron beam density in a well-defined fiducial volume in the beam and by the accuracy of absolute measurement of the neutron decay products (protons or electrons) count rate.

In the ultracold neutron (UCN) storage method the neutron lifetime is measured by counting surviving neutrons in a magnetic or material trap as a function of time. No absolute measurement is necessary in this case but serious problem arises in view of UCN losses in traps. In the UCN magnetic storage experiments the problem is in possible neutron spin-flip followed by its escape from the magnetic storage volume. Another problem is the marginal neutron trapping due to specular reflection of superbarrier neutrons from the ideally smooth magnetic mirror leading to appearance of quasi-bound neutron orbits.

In material traps the main problem is to account properly for the UCN losses in their collisions with the surface of a material confinement cavity. One tries to minimize these losses choosing the materials with the lowest neutron capture and suppressing the neutron upscattering by lowering the trap temperature.

The value of necessary corrections and systematic errors in inferring the neutron lifetime from the UCN storage data depend on these losses.

The present experimental data base for the free neutron decay is large but contains significant discrepancy between data. The Table shows the values of the neutron lifetime obtained in the beam experiments and in the UCN storage experiments. Only the results with uncertainties not exceeding $10 \mathrm{~s}$ were taken 
into consideration.

Results of the neutron lifetime measurements in the beam experiments and in the UCN storage experiments

Beam experiments

$891 \pm 9$ (1988, P. E. Spivak) [4]

*893.6 $\pm 3.8 \pm 3.7$ (1990, J. Byrne et al.) [5]

$889.2 \pm 3.0 \pm 3.8$ (1996, J. Byrne et al.) [6]

*886.8 $\pm 1.2 \pm 3.2$ (2003, M. S. Dewey et al.) [7]

*886.6 $\pm 1.2 \pm 3.2$ (2004, J. S. Nico et al.) [8]

$886.3 \pm 1.2 \pm 3.2$ (2005, M. S. Dewey et al.) [9]
Storage experiments

$877 \pm 10$ (1989, F. Anton et al.) [10]

$870 \pm 8$ (1989, A. G. Kharitonov et al.) [11]

$887.6 \pm 3.0$ (1989, W. Mampe et al.) [12]

888.4 \pm 3.3 (1992, V. Nesvizhevsky et al.) [13]

$882.6 \pm 2.7$ (1993, W. Mampe et al.) [14]

$885.4 \pm 0.9 \pm 0.4$ (2000, S. Arzumanov et al.) [15]

881. \pm 3.0 (2000, A. Pichlmaier et al.) [16]

$878.5 \pm 0.7 \pm 0.3$ (2004, A. Serebrov et al.) [17]

* 874.6 $6_{-1.6}^{+4}$ (2007, V. Ezhov et al.) [18]

$878.2 \pm 1.9$ (2009, V.Ezhov et al.) [19]
Averaged value

$887.6 \pm 2.7$
Average value without [17] and [19]

$885 . \pm 0.82$

Average value including [17] and [19]

$881.3 \pm 0.53$

The data marked with the * are not used for obtaining the average values, only the last results of the corresponding experiment are taken into consideration.

The world average value [20] based on $[4,6,9,12,13,14,15]$ is $885.7 \pm 0.8 \mathrm{~s}$.

It is seen that the most precise UCN storage results may be divided in two groups: one - Refs.[1215] and another - Refs.[17,19], with the work [16] between these groups. In view of this disagreement between UCN storage measurements and some difference between the results of the beam and the UCN storage experiments, new precision measurements of the neutron lifetime by different methods are desirable. It is essential that in the UCN storage experiments the total neutron disappearance probability is measured, whereas the beam experiments are only sensitive to the neutron beta decay.

\section{DIFFERENT FORMS OF THE UCN MATERIAL TRAPS}

Different geometries (Fig. 1) of storage volume are considered here: cylindrical with vertically and horizontally directed axis of the cylinder in respect to the Earth gravitational field, spherical, paraboloidal, 

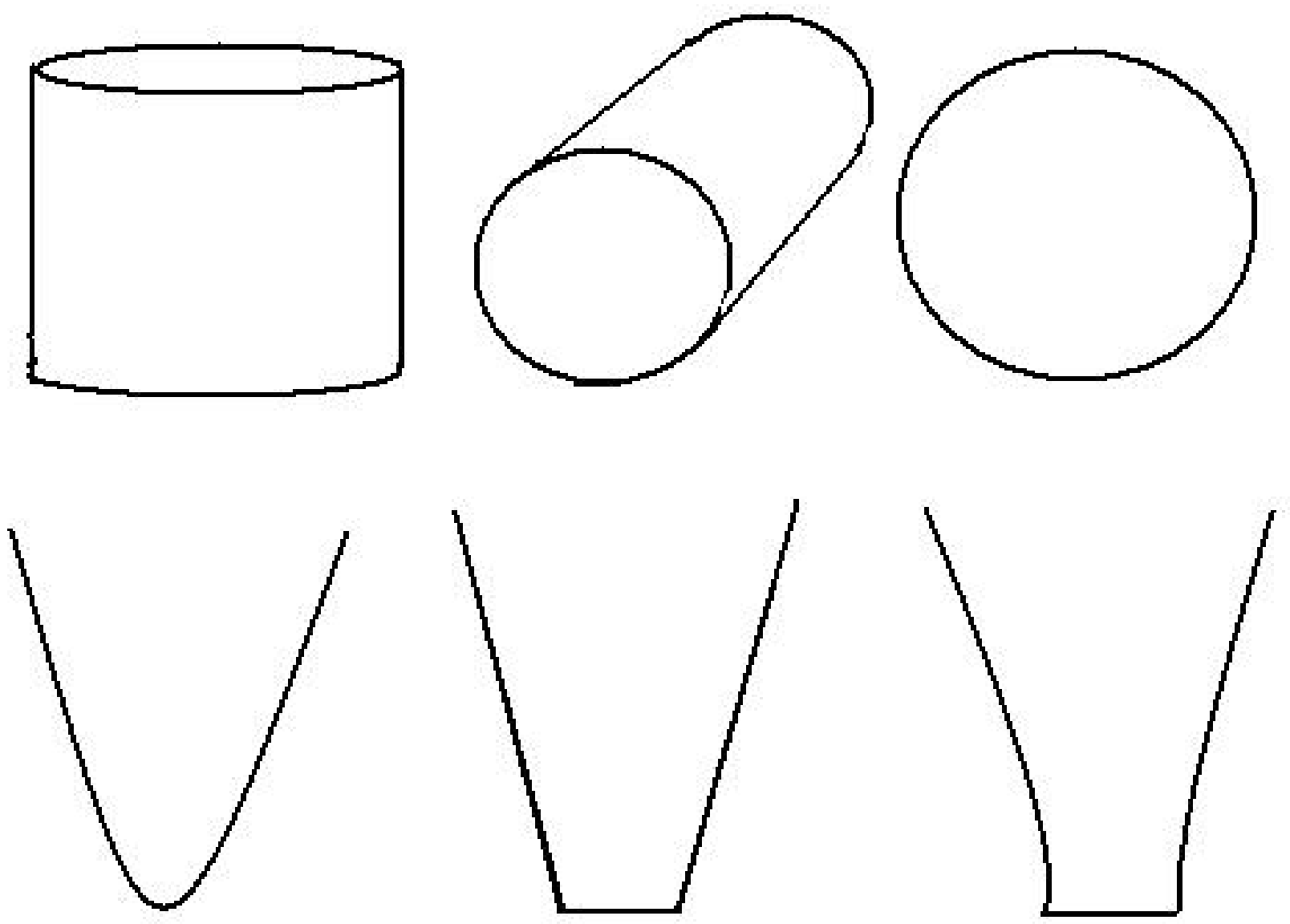

Figure 1: The forms of the UCN traps. 
conical, and vase. The quasi-spherical and horizontal cylinder geometries have been used in the neutron lifetime measurements $[11,13,17]$, the vertical cylinder has been used in the first neutron lifetime experiments with UCN [21], the conical, vase and paraboloidal forms have never been used and are considered for comparison.

In the presence of gravity, for equilibrium distribution of ultracold neutrons in the available phase space in a cavity, the neutron loss rate $\left(\mathrm{s}^{-1}\right)$ is determined by the equation (we follow here the approach of [22]):

$$
d \int \varrho(\mathbf{r}, \mathbf{v}, t) d^{3} \mathbf{r} d^{3} \mathbf{v}=-\int \mu(\mathbf{v})\left(\mathbf{v n}_{\mathbf{S}}\right) \varrho(\mathbf{r}, \mathbf{v}, t) d^{3} \mathbf{v} d S d t .
$$

Here, $\varrho(\mathbf{r}, \mathbf{v}, t)$ is the UCN phase space density, $\mathbf{n}_{\mathbf{S}}$ is the normal to the surface at the point of neutron collision with the wall. The neutron loss probability per one collision $\mu(v)$, for isotropic neutron incidence, according to the accepted formalism is:

$$
\bar{\mu}(v)=2 \eta \frac{\kappa(y)}{y^{2}}, \quad \kappa(y)=\arcsin y-y \sqrt{1-y^{2}}, \quad y=v / v_{b}=\sqrt{E / V},
$$

where $v$ and $E$ are the neutron velocity and energy, respectively, $v_{b}$ is the neutron boundary velocity of the cavity, $\eta$ is the UCN loss coefficient determined by the properties of the wall material:

$$
\eta=-\operatorname{Im} U / \operatorname{Re} U, \quad U=\left(\hbar^{2} / 2 m\right) 4 \pi \sum_{i} N_{i} b_{i}, \quad V=\operatorname{Re} U, \quad \operatorname{Im} b=-\sigma / 2 \lambda .
$$

Here the complex potential $U$ describes UCN interaction with walls, $m$ is the neutron mass, $N_{i}$ is the number density of nuclei of type $i$ in the material, $b_{i}$ is the corresponding coherent scattering length on a bound nucleus of the wall, and $\sigma$ is the cross section of inelastic processes (capture plus upscattering) for neutrons with wavelength $\lambda$.

The neutron density depends on the height coordinate $z$ defined relative to the lowest point of a cavity:

$$
\varrho(z, v)=c \delta\left(v_{0}^{2}-2 g z-v^{2}\right),
$$

where $c$ is the normalization constant, $v_{0}$ and $\varrho(0, v)=c \delta\left(v_{0}^{2}-v^{2}\right)$ are the neutron velocity and the neutron density at the bottom of the trap, $g=9.80665 \mathrm{~m} / \mathrm{s}^{2}$ is gravitational acceleration.

Solution of Eq. (1) gives the UCN loss rate for different forms of the traps.

General expression for the UCN loss probability $\left(\mathrm{s}^{-1}\right)$ of the neutron with velocity $v$ at the bottom of the cavity is

$$
\tau^{-1}(y)=\eta \gamma(y) ; \quad \gamma(y)=v_{b} \frac{S(y)}{V(y)},
$$

where $S(y)$ is the surface loss term and $V(y)$ is the effective volume.

In a cylindrical trap with horizontal axis the expressions for $S(y)$ and $V(y)$ are:

$$
S(y)=S_{\mathrm{cyl}}(y)+2 S_{\mathrm{pl}}(y), \quad S_{\mathrm{cyl}}(y)=R H \int_{0}^{\varphi_{0}} \kappa\left(\sqrt{y^{2}-2 g R(1-\cos \varphi) / v_{b}^{2}}\right) d \varphi
$$




$$
\begin{aligned}
S_{\mathrm{pl}}(y) & =\int_{0}^{z_{0}} \kappa\left(\sqrt{y^{2}-2 g z / v_{b}^{2}}\right) \sqrt{2 R z-z^{2}} d z, \\
V(y) & =2 H \int_{0}^{z_{0}} \sqrt{y^{2}-2 g z / v_{b}^{2}} \sqrt{2 R z-z^{2}} d z,
\end{aligned}
$$

where $S_{\text {cyl }}(y)$ is the loss contribution of the cylinder surface, $S_{\mathrm{pl}}(y)$ is the loss contribution of each of two plane surfaces, $\varphi_{0}=\arccos \left(1-\frac{\left(y v_{b}\right)^{2}}{2 g R}\right), z_{0}=\left(y v_{b}\right)^{2} / 2 g, R$ and $H$ are the radius and the length of the horizontal cylinder, respectively.

For a spherical trap of radius $R$ these expressions have the form:

$$
S(y)=\pi R \int_{0}^{z_{0}} \kappa\left(\sqrt{y^{2}-2 g z / v_{b}^{2}}\right) d z, \quad V(y)=\pi \int_{0}^{z_{0}} \sqrt{y^{2}-2 g z / v_{b}^{2}}\left(2 R z-z^{2}\right) d z .
$$

For a conical cavity with the angle $\theta$ and flat bottom of radius $r_{0}$ the loss rate is determined by:

$$
\begin{array}{r}
S(y)=S_{\mathrm{con}}(y)+S_{\mathrm{bot}}(y), \quad S_{\mathrm{con}}(y)=\pi \int_{0}^{z_{0}} \kappa\left(\sqrt{y^{2}-2 g z / v_{b}^{2}}\right) \frac{z \tan (\theta)}{\cos \theta} d z, \\
S_{\mathrm{bot}}(y)=\frac{\pi r_{0}^{2}}{2} \kappa(y), \quad V(y)=\pi \int_{0}^{z_{0}} \sqrt{y^{2}-2 g z / v_{b}^{2}}(z \tan \theta)^{2} d z,
\end{array}
$$

where $S_{\text {con }}(y)$ is the conical surface losses and $S_{\text {bot }}(y)$ is the bottom surface losses.

For a vertical cylinder trap with the radius $R$ the loss rate is determined by:

$$
\begin{array}{r}
S(y)=S_{\mathrm{cyl}}(y)+S_{\mathrm{bot}}(y), \quad S_{\mathrm{cyl}}(y)=\pi R \int_{0}^{z_{0}} \kappa\left(\sqrt{y^{2}-2 g z / v_{b}^{2}}\right) d z, \\
S_{\mathrm{bot}}(y)=\frac{\pi R^{2}}{2} \kappa(y), \quad V(y)=\pi R^{2} \int_{0}^{z_{0}} \sqrt{y^{2}-2 g z / v_{b}^{2}} d z,
\end{array}
$$

where $S_{\text {cyl }}(y)$ is the contribution of the cylinder surface.

For a paraboloidal cavity $\left(z=r^{2} / R\right)$ the loss rate is determined by

$$
S(y)=\pi \int_{0}^{z_{0}} \kappa\left(\sqrt{y^{2}-2 g z / v_{b}^{2}}\right)\left(R z+\frac{R^{2}}{4}\right) d z, \quad V(y)=\pi R \int_{0}^{z_{0}} \sqrt{y^{2}-2 g z / v_{b}^{2}} z d z .
$$

For a cavity in the form of a vase $\left(z=(R r)^{1 / n}-b\right)$ with the flat bottom of radius $r_{0}$ and radius $r_{m}$ at the height $H$ the surface loss and the effective volume are:

$$
\begin{array}{r}
S(y)=S_{\text {vase }}(y)+S_{\mathrm{bot}}(y), \\
S_{\text {vase }}(y)=\frac{\pi}{R} \int_{0}^{z_{0}} \kappa\left(\sqrt{y^{2}-2 g z / v_{b}^{2}}\right)(z+b)^{n}\left[1+\frac{n^{2}(z+p)^{2(n-1)}}{R^{2}}\right] d z, \\
S_{\mathrm{bot}}(y)=\frac{\pi r_{0}^{2}}{2} \kappa(y), \\
V(y)=\frac{\pi}{R^{2}} \int_{0}^{z_{0}}(z+p)^{2 n} \sqrt{y^{2}-2 g z / v_{b}^{2}} d z .
\end{array}
$$

Here, $R=H^{n} /\left(r_{m}^{1 / n}-r_{0}^{1 / n}\right)$ and $p=\left(R r_{0}\right)^{1 / n}$. 


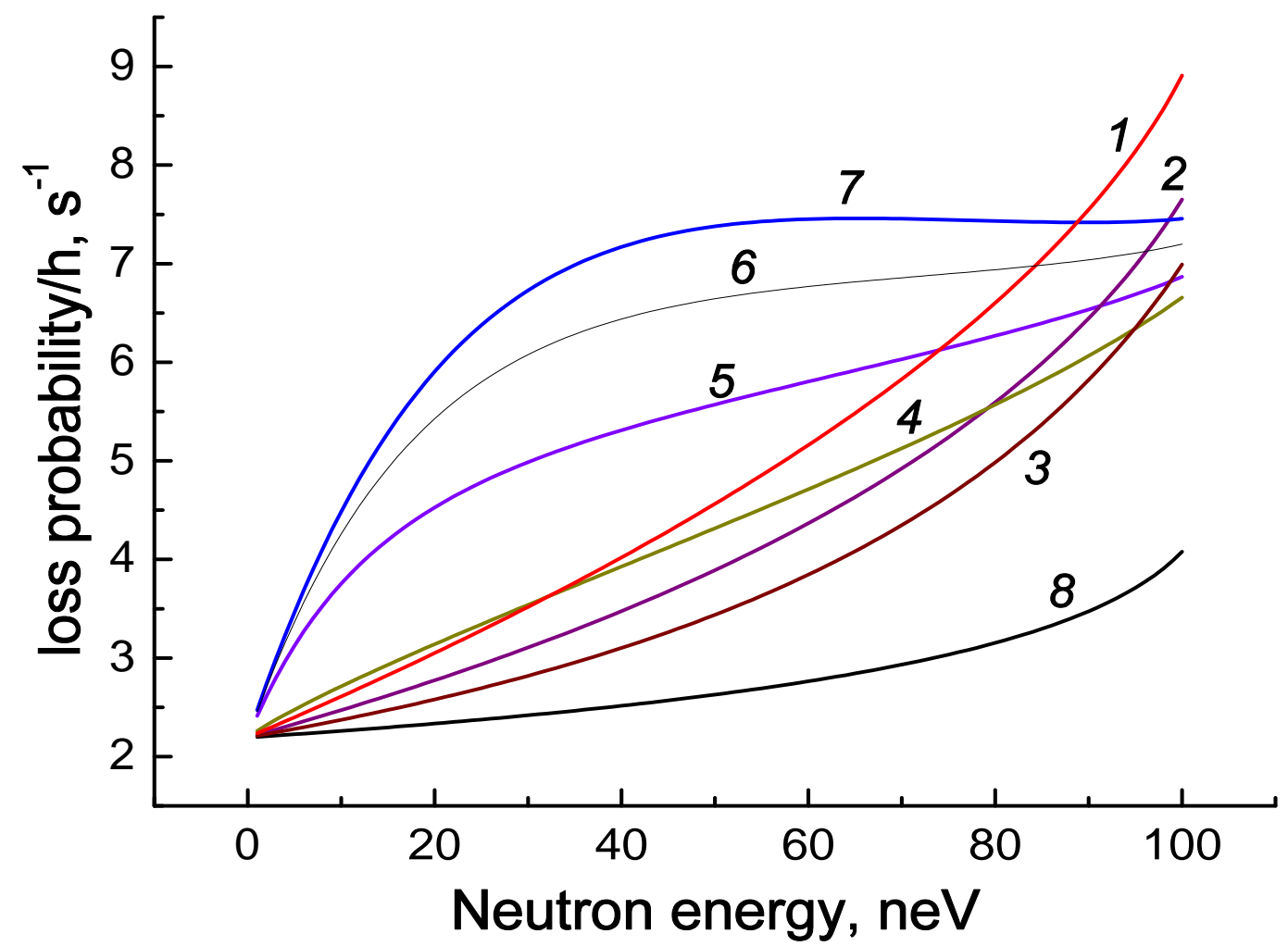

Figure 2: The reduced UCN loss probability $\left(\mathrm{s}^{-1}\right)$ as a function of neutron energy for different forms of neutron traps: 1 - vertical cylinder, $\mathrm{R}=50 \mathrm{~cm}, 2$ - horizontal cylinder with radius of $50 \mathrm{~cm}$ and length $100 \mathrm{~cm}, 3$ - sphere, $\mathrm{R}=50 \mathrm{~cm}, 4$ - paraboloid $z=r^{2} / 25,5$ - truncated cone, $\theta=\pi / 6$, the bottom radius $r_{0}=5 \mathrm{~cm}, 6$ - truncated cone, $\theta=\pi / 4$, the bottom radius $r_{0}=5 \mathrm{~cm}, 7-$ vase cavity $(z=\sqrt{R r}-p)$, with bottom radius of $5 \mathrm{~cm}$, top radius of $50 \mathrm{~cm}$ and height of $100 \mathrm{~cm}, 8-$ infinite plane. 
Fig. 2 shows the reduced (the loss coefficient $\eta=1$ ) UCN loss probability $\left(\mathrm{s}^{-1}\right)$ as a function of neutron energy at the bottom of the traps of different form: cylindrical with vertically and horizontally directed axis of the cylinder in respect to the Earth gravitational field, spherical, paraboloidal, conical, and vase. For comparison the loss probability is shown also for neutrons bouncing at the infinite horizontal plane.

All results are shown for $v_{b}=447 \mathrm{~cm} / \mathrm{s}$ - the boundary velocity for the fully fluorinated polyether oils (perfluoropolyethers - PFPE) used in the recent UCN lifetime measurement and having good neutron reflection properties [12,16,17].

As is seen, there is significant difference in the values and energy dependence of the neutron losses in the traps of similar size but different form. The traps expanding to the top, especially conical ones and in the form of vase, have low energy dependence of losses in a wide energy range.

\section{EXTRACTION OF THE NEUTRON DECAY CONSTANT}

The total loss rate $\tau_{\mathrm{st}}^{-1}$ of neutrons stored in a trap is determined by the neutron decay constant $\tau_{\text {decay }}^{-1}$ and the wall loss rate $\tau_{\text {loss }}^{-1}$ :

$$
\tau_{\text {st }}^{-1}=\tau_{\text {decay }}^{-1}+\tau_{\text {loss }}^{-1}
$$

There are two methods of extracting the neutron decay constant from storage measurements in the presence of losses.

The first one consists in exclusion of the UCN surface losses from the experimental $\tau_{\mathrm{st}}^{-1}$ using several traps with the same UCN loss coefficient $\eta$ but with different UCN free path lengths between collisions with walls:

$$
\tau_{\text {st }, i}^{-1}=\tau_{\text {decay }}^{-1}+\eta \gamma_{i},
$$

where $\tau_{\mathrm{st}, i}^{-1}$ and $\gamma_{i}$ are the inverse experimental storage time and the calculated reduced (without $\eta$ ) loss in $i$-th trap, respectively.

The first method is realized introducing additional surface at the same storage volume [21] or using the traps of same form but different size (size extrapolation) [12,14,16,17].

For extrapolation the measured $\tau_{\mathrm{st}, i}^{-1}$ are plotted against calculated $\gamma_{i}$ or against calculated inverse free path length $\lambda^{-1}$ between neutron collisions with walls to obtain the intercept with the $\gamma=0$ - axis.

In the second method the UCN storage is performed in the same trap but with a sequence of different neutron energy spectra - the energy extrapolation method $[12,13,17]$.

Sharp energy dependence of losses is essential for extracting the neutron decay constant from the storage data by the energy extrapolation method. Weak energy dependence of losses on the neutron energy gives (for example in the energy range from 30 to $100 \mathrm{neV}$ for the curve 7 at Fig. 2) relative insensitivity of the storage time to the neutron spectrum. Conical and vase traps with their weak energy dependence of losses can be used more efficiently with the size extrapolation.

One key systematic effect, that may arise when using the size extrapolation method with two or more neutron traps, is possible difference in the value of neutron losses at the surface of different traps. On 


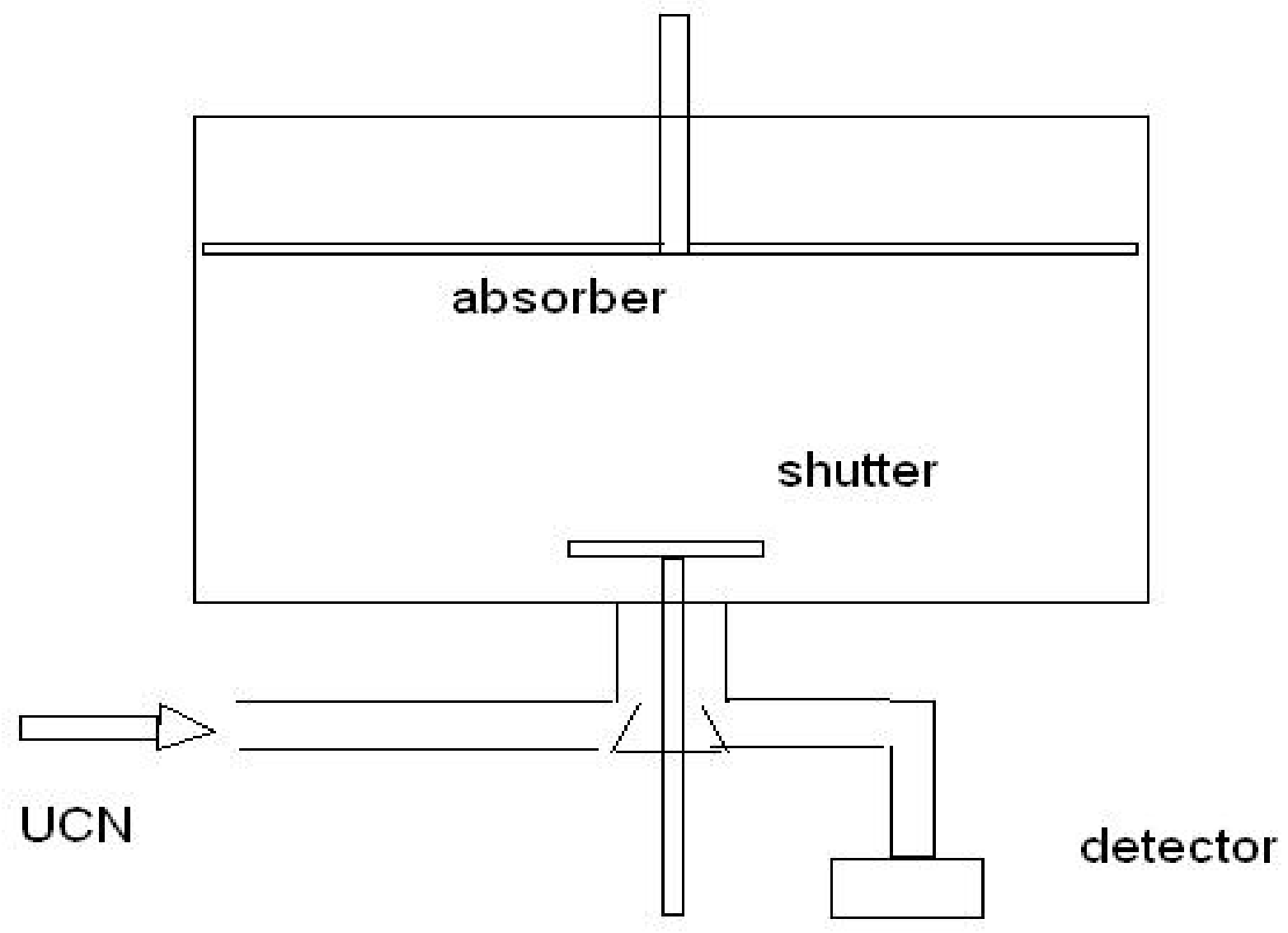

Figure 3: The scheme of the UCN neutron lifetime measurement.

the other hand in the energy extrapolation method, applied to the UCN storage data obtained in the same neutron trap, the homogeneity of the neutron reflecting coverage of the trap surface is not less important.

Contrary to the size extrapolation the energy extrapolation needs more precise information on the realistic energy dependence of collision neutron losses. The energy dependence of UCN losses has been measured twice: first for copper surface in [23] and then with better precision for the Fomblin oil and grease surfaces in [24]. No deviation has been found in both measurements from the energy dependence of Eq. (2) except for the increase in losses for the neutrons with energy in the vicinity of the boundary potential of Fomblin oil [24]. This could be caused by additional small UCN upscattering by liquid surface modes of Fomblin.

Actually, it is better to combine both methods of extrapolation in the neutron lifetime experiment in material traps [17].

The scheme considered here in more details is shown in Fig. 3. The storage measurements are performed in two storage traps of different size (e.g., two vertical cylinders with the diameters of 50 and $100 \mathrm{~cm}$ ) for a sequence of energy spectra of stored neutrons: with the upper energy cut-off from the lowest possible, say $10 \mathrm{neV}$, up to the largest possible for the used material, e.g. $\sim 100 \mathrm{neV}$ for 
fluoropolymers. At small (100-200 s) storage time the initial integral spectra of stored neutrons is measured, at long storage ( $\sim 1000-2000 \mathrm{~s})$ the measured storage time comprises the neutron decay as well as the energy dependent losses.

\section{THE UCN SPECTRA FORMATION}

Sharp cutoff in the spectrum may be obtained with the help of a neutron absorber placed horizontally in the upper part of the storage volume in the cavity. The quality of the UCN absorber is determined by the height of the potential of the absorbing material. Traditionally, polyethylene $\left(\mathrm{CH}_{2}\right)_{n}$ with its comparatively low negative potential for neutrons $U\left(\mathrm{CH}_{2}\right)_{n} \approx-9 \mathrm{neV}$, was considered as an "ideal" absorber.

Metal absorbers with low or negative scattering length like Ti or V may be not the best choice as the most effective UCN absorbers in view of oxidation of its surface. Even very thin - dozens $\AA$ - oxide film forming positive potential barrier entails significant UCN reflection from the surface. Fig. 4 shows the neutron energy dependence of reflectivity at isotropic neutron incidence for polyethylene together with two other possible materials with good compensation of positive and negative coherent scattering lengths of nuclei - glyceride of melyssinic acid $\mathrm{C}_{96} \mathrm{H}_{188} \mathrm{O}_{6}$ with the height of the potential $U=-3.3 \mathrm{neV}$ and Zirconium hydride $\mathrm{ZrH}_{2}$ with almost perfect compensation of the scattering lengths: $U=-0.325 \mathrm{neV}$.

The characteristic "cleaning time" - the loss time for UCN with the energies exceeding the cutoff energy determined by the height of the neutron absorber, may be calculated in the same formalism as was used for calculations the neutron loss rate in traps (Eqs. (1)-(5)).

The absorption probability of the neutron with the energy $E$ incident on a surface with an ideal step function profile at the angle $\theta$ in respect to the surface normal is

$$
T\left(E, \theta, E_{b}\right)=\frac{4 \cos \theta \sqrt{\cos ^{2} \theta-\left(E_{b} / E\right)}}{\left(\cos \theta+\sqrt{\cos ^{2} \theta-\left(E_{b} / E\right)}\right)^{2}},
$$

where $E_{b}$ is the boundary energy of the absorber.

The expression for the absorption time constant for a vertical cylinder trap with the absorber at the height $H$ with respect to the bottom of the cylinder, has the view:

$$
\tau_{\text {clean }}(v, H)=\frac{K_{3}}{K_{1}}
$$

where

$$
\begin{gathered}
K_{1}=\left(v^{2}-2 g H\right) \int_{0}^{\pi / 2} \frac{4 \sin (2 \theta) \cos \theta\left(\cos ^{2} \theta \mp v_{a}^{2} /\left(v^{2}-2 g H\right)\right)^{1 / 2}}{\left[\cos \theta+\left(\cos ^{2} \theta \mp v_{a}^{2} /\left(v^{2}-2 g H\right)\right)^{1 / 2}\right]^{2}} d \theta, \\
K_{3}=\frac{v^{3}-\left(v^{2}-2 g H\right)^{3 / 2}}{3 g},
\end{gathered}
$$

$v_{a}^{2}=2\left|E_{b}\right| / m$, and the signs "-" and "+" correspond to positive and negative boundary energies of the absorber respectively. In the case of positive boundary energy of the absorber the integration over angle is performed over angles when $\cos ^{2}(\theta)>v_{a}^{2} /\left(v^{2}-2 g H\right)$. The Eqs. (16) are valid when $v^{2}>2 g H$. 


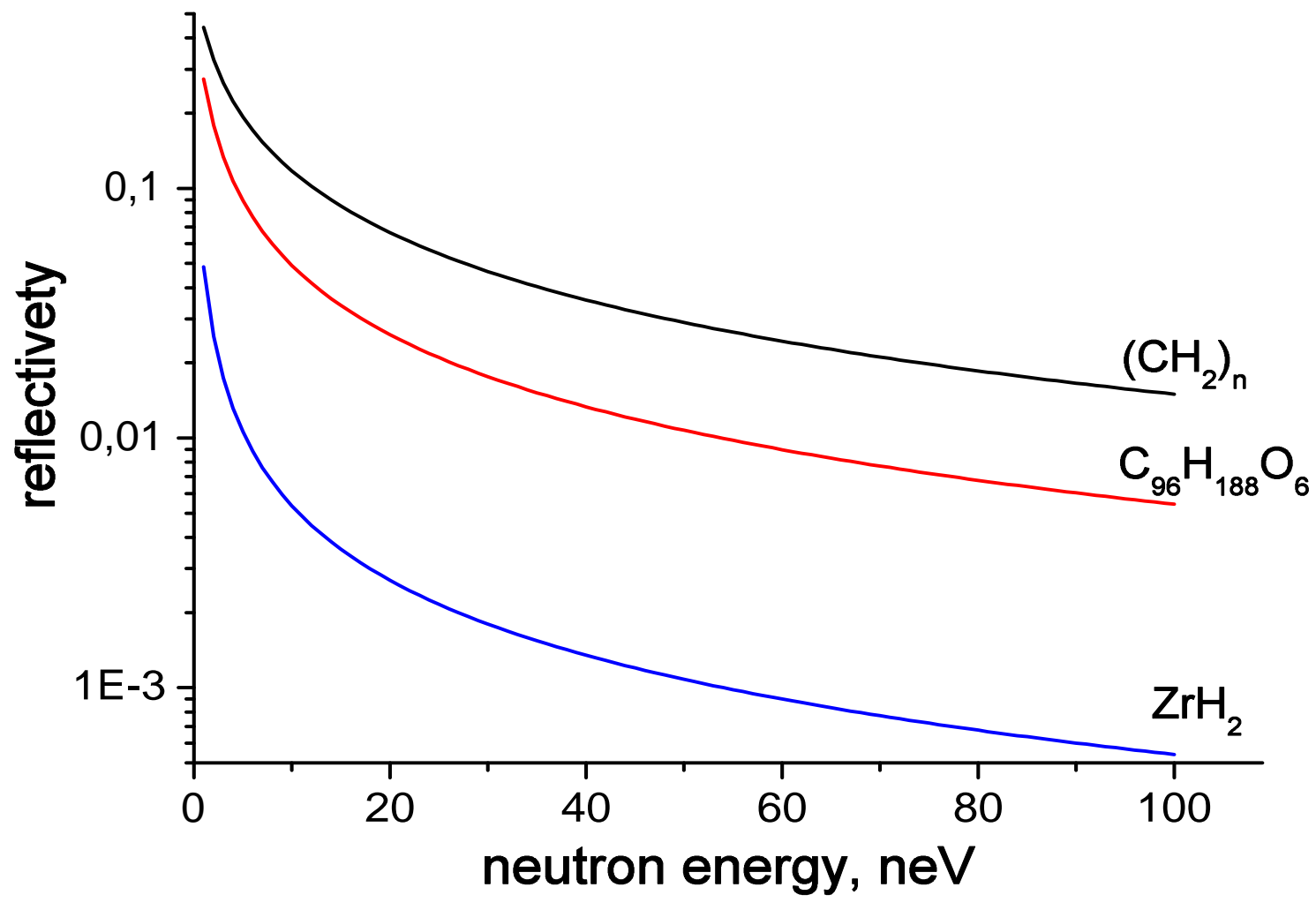

Figure 4: The energy dependence of the UCN reflection probability from polyethylene $\left(\mathrm{CH}_{2}\right)_{n}$, glyceride of melissynic acid $\mathrm{C}_{96} \mathrm{H}_{188} \mathrm{O}_{6}$ and Zirconium hydride $\mathrm{ZrH}_{2}$ at isotropic neutron incidence. 


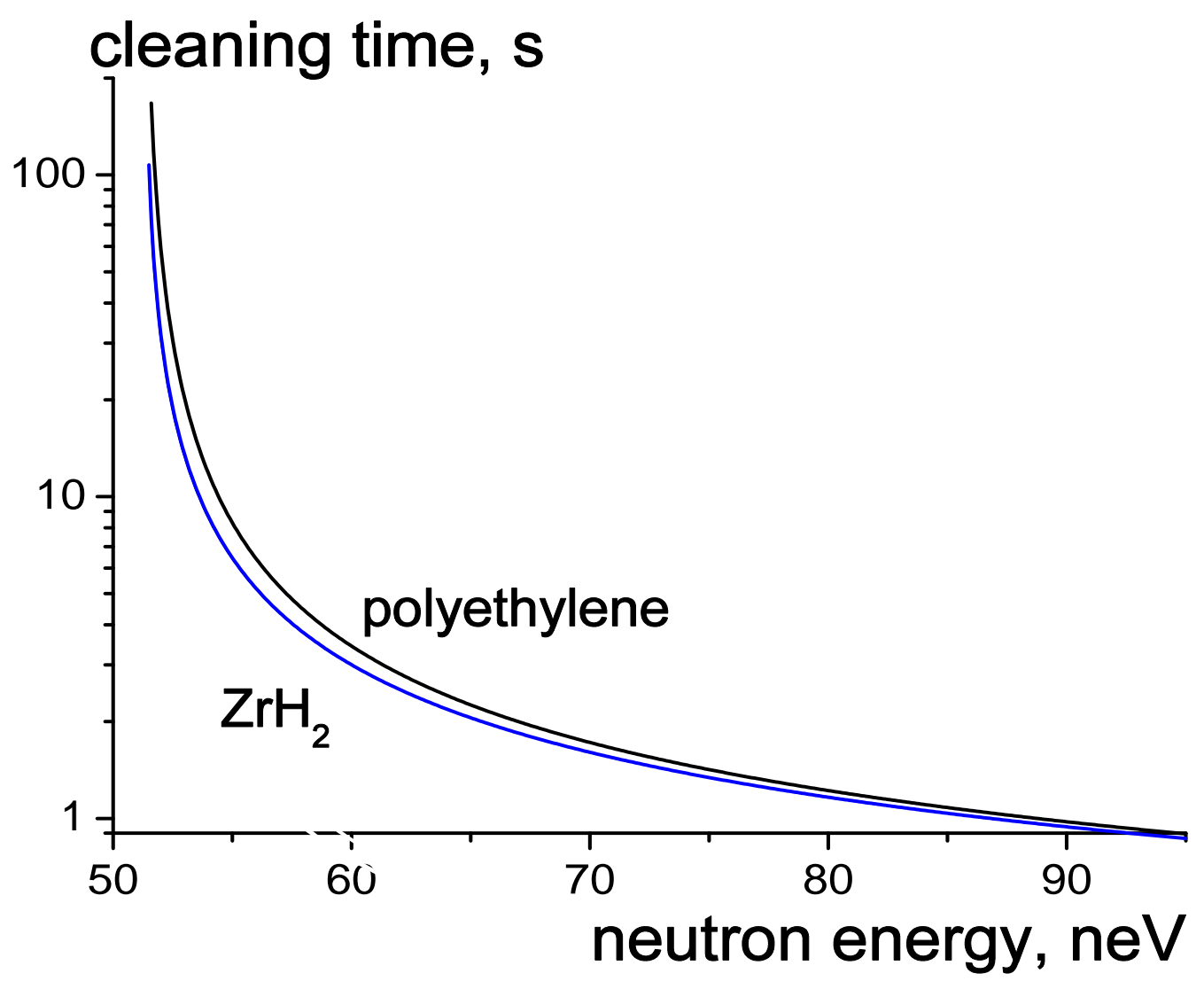

Figure 5: The energy dependence of the UCN cleaning time in a vertical cylinder with an absorber at $\mathrm{H}=50 \mathrm{~cm}$ for polyethylene, $\mathrm{ZrH}_{2}$ and an ideal absorber. The latter practically coincides with an ideal absorber.

Fig. 5 shows the energy dependence of the "cleaning time" for three UCN absorbers: polyethylene, $\mathrm{ZrH}_{2}$, and an ideal absorber (100\% loss probability for all neutron energies) in the vertical cylinder with the absorber placed at the height $H=50 \mathrm{~cm}$. The "cleaning times" for $\mathrm{ZrH}_{2}$ and an ideal absorber are practically the same.

It is seen that the polyethylene absorber is generally not seriously inferior in respect to an ideal one except for the energy range close to the cutoff energy, where the cleaning time for the polyethylene may exceed several times the cleaning time for an ideal absorber.

The time needed for cleaning spectrum should not be too large in view of additional neutron losses during cleaning process.

In [25] it was proposed to construct a new gravitational trap similar to described in [17] - rotating horizontal cylinder with the radius of $120 \mathrm{~cm}$. It is obvious that the spectrum cleaning time for such a trap may be very large.

The UCN escape from a horizontal cylinder having the longitudinal hole with the angular width $2 \theta_{0}$ 


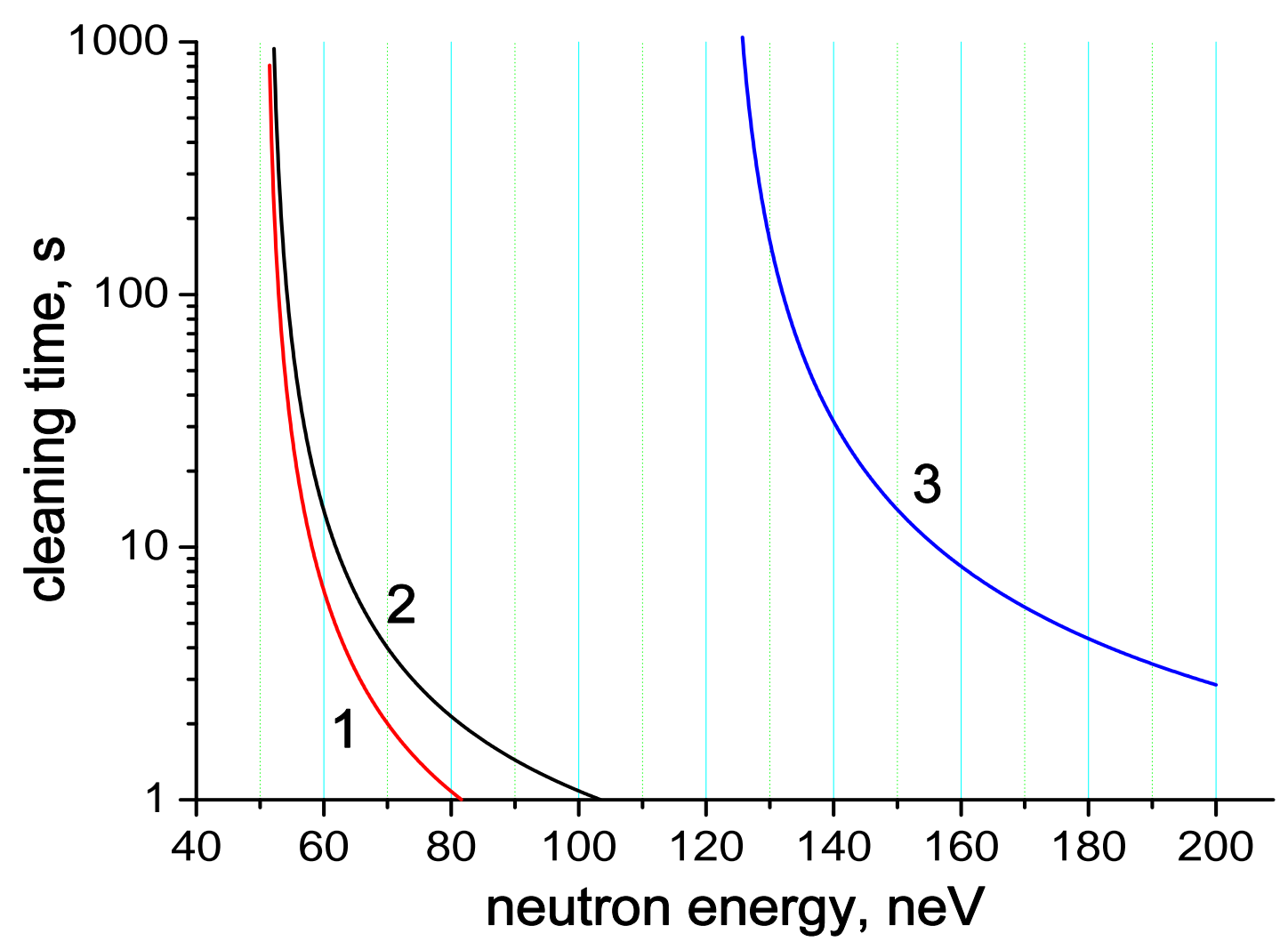

Figure 6: Energy dependence of the calculated UCN escape time from horizontal cylinders: $1-$ the diameter of $50 \mathrm{~cm}$ with the hole in the upper side with $2 \theta_{0}=0.4 \mathrm{rad}, 2-$ the half-cylinder of radius 50 $\mathrm{cm}, 3$ - the half-cylinder of radius $120 \mathrm{~cm}$.

in the upper side of the cylinder may be imitated by placing the flat vertical ideal absorber as an upward directed prolongation of the hole. The escape rate is calculated according:

$$
\begin{array}{r}
f=\frac{S(v)}{V_{1}(v)+V_{2}(v)}, \quad S(v)=\frac{2 R \cos \theta_{0}+H}{2} \int_{R\left(1+\cos \theta_{0}\right)}^{v^{2} / 2 g}\left(v^{2}-2 g z\right) d z \\
V_{1}(v)=2 H R^{2} \int_{0}^{\pi-\theta_{0}} \sin ^{2} \theta \sqrt{v^{2}-2 g R(1-\cos \theta)} d \theta \\
V_{2}(v)=2 H R \int_{R\left(1+\cos \theta_{0}\right)}^{v^{2} / 2 g} \sqrt{v^{2}-2 g z} d z,
\end{array}
$$

where $S(v)$ is the loss term due to collisions with the absorber, $V_{1}(v)$ and $V_{2}(v)$ are effective volumes of the assumed to be non-absorbing in this case cylinder and the absorbing prolongation, respectively.

The neutron escape time from horizontal cylinders is shown in Fig. 6 for three cases: $2 \theta=0.4 \mathrm{rad}$, the cylinder radius $25 \mathrm{~cm}$, and $2 \theta_{0}=\pi$ (open half-cylinder), the cylinder radius $50 \mathrm{~cm}$ and $120 \mathrm{~cm}$.

\section{THE SIMULATION OF THE EXPERIMENTS}




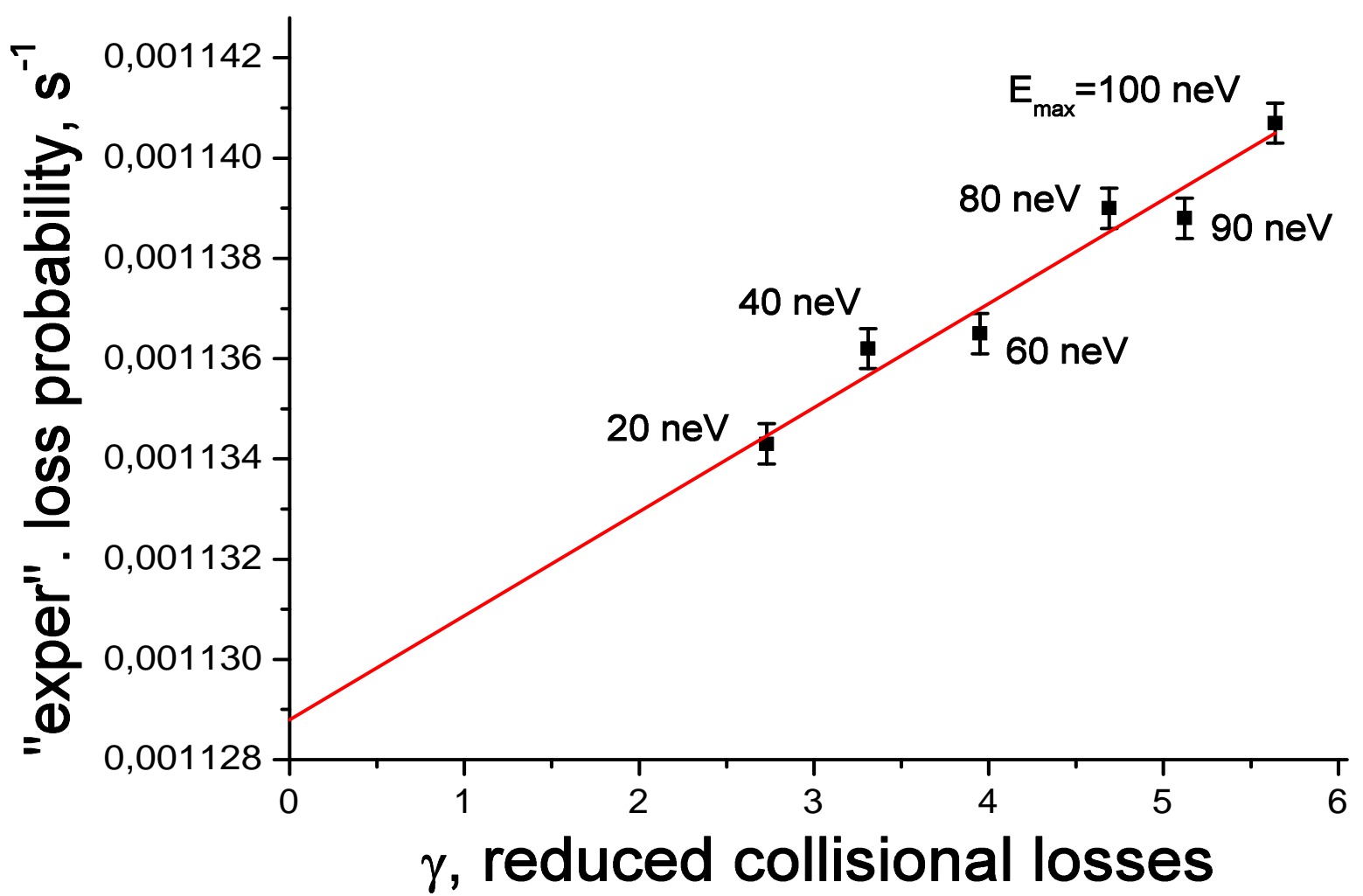

Figure 7: The results of the simulation of the neutron lifetime experiment in the vertical cylinder trap with radius $50 \mathrm{~cm}$. The simulation of the neutron storage was performed for the Maxwellian spectra with the energy cut-offs at 20,40,60,80, 90 and $100 \mathrm{neV}$. The neutron decay lifetime was $885.7 \mathrm{~s}$.

The results of simulation of the experiment with the UCN energy extrapolation is illustrated in Fig. 7 for the vertical cylinder trap of the radius $50 \mathrm{~cm}$.

The storage times and the values of collision losses are calculated for 6 different cut-offs of the initial stored UCN spectrum: from $20 \mathrm{neV}$ to $100 \mathrm{neV}$. The loss coefficient was taken $\eta=2 \times 10^{-6}$ [26,17], the neutron decay lifetime $885.7 \mathrm{~s}$ [20], the neutron loss function has the form of Eq. (2), and it was assumed that initial neutron spectrum at each measurement is the Maxwell-like tail with perfect cut-off at the corresponding maximum energy. It was assumed that the storage times were measured with a statistical uncertainty of $4 \times 10^{-4}$ (100 and $1000 \mathrm{~s}$ measurements, each with $10^{7}$ counts), and were distributed randomly. To reach this statistical precision needs $\sim 530$ cycles of fillings the neutron storage cavity if the number of stored neutrons in full spectrum with the $E_{\max }=100 \mathrm{neV}$ is $10^{6}$ per filling and linearly depends on the cutoff energy.

In reality extrapolation of storage data to zero losses takes place in condition of non-complete information about the energy spectrum. The incident integral UCN spectrum is measured at small storage 


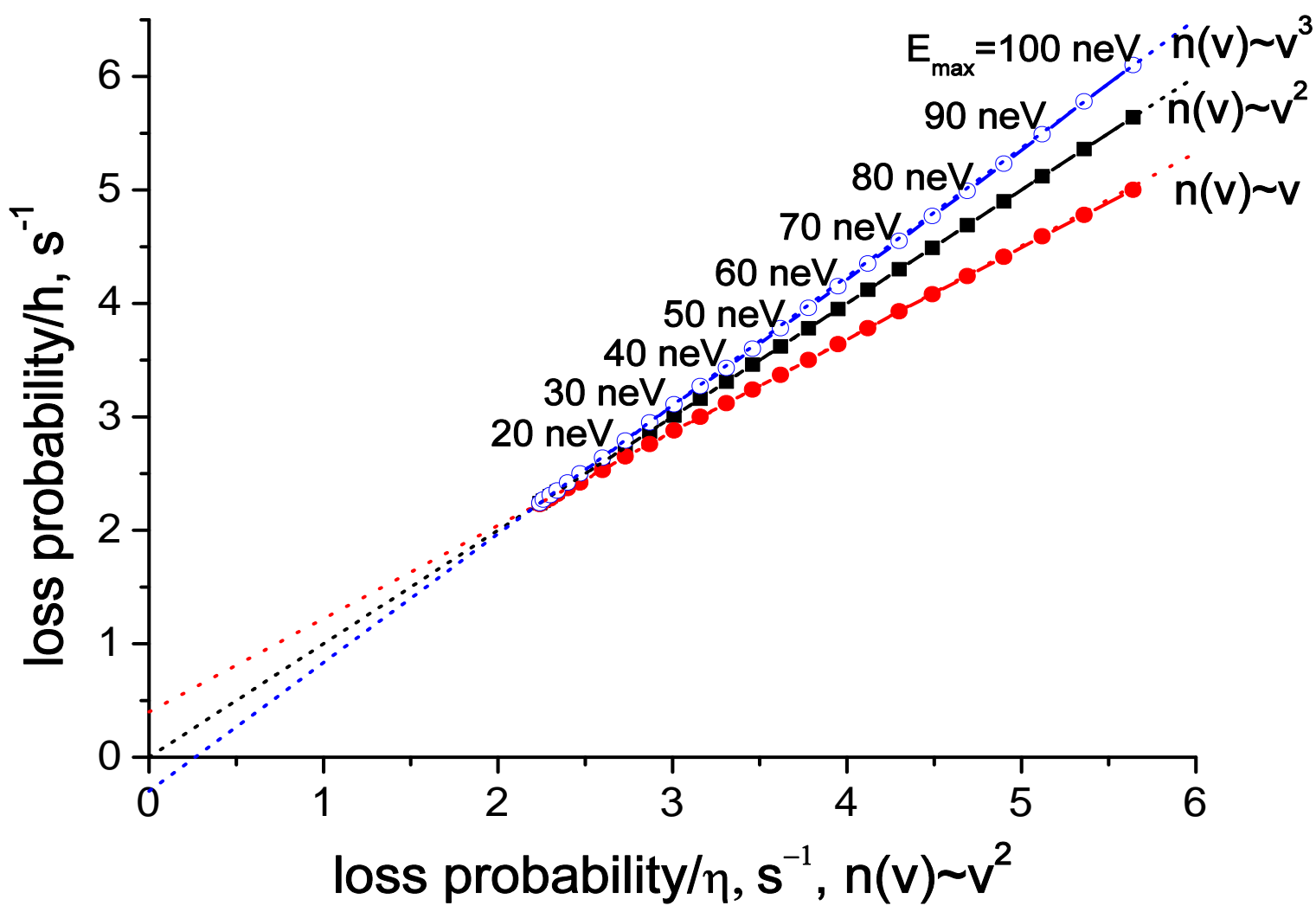

Figure 8: The reduced UCN loss probability in the vertical cylinder trap for different UCN spectra as a function of reduced UCN loss probability for the Maxwellian tail spectrum in an assumption of the standard reflection law of Eq. (2).

time by changing the height of the absorber. But precision of this measurement is not perfect and the incident UCN spectrum in the trap may differ from the Maxwell-like form. Therefore extrapolation to zero losses in an assumption of of the Maxwell-like spectrum may incur errors.

Fig. 8 shows the calculated loss rate for different UCN spectra as a function of the calculated loss rate for the Maxwell-like spectrum.

The extrapolated losses to zero energy differ from zero in the cases, when the spectra are not Maxwellian, by the value $\sim 10^{-6} \mathrm{~s}^{-1} \approx 10^{-3} \tau_{n}^{-1}$ if the $\eta=2 \times 10^{-6}$. It follows from this preliminary estimate that the measurement of the incident UCN spectra with $5 \%$ precision will decrease this correction to the level $5 \times 10^{-5} \tau^{-1}$.

More serious correction may appear if the real dependence of UCN losses on the neutron energy differs from the form of Eq. (2) calculated for the ideal step potential. Analysis shows that the UCN loss energy dependence should be determined in this experiment with the precision no less than $5 \%$.

Fig. 9 shows the calculated UCN loss rate for the cases when there is $5 \%$ admixture to the standard 


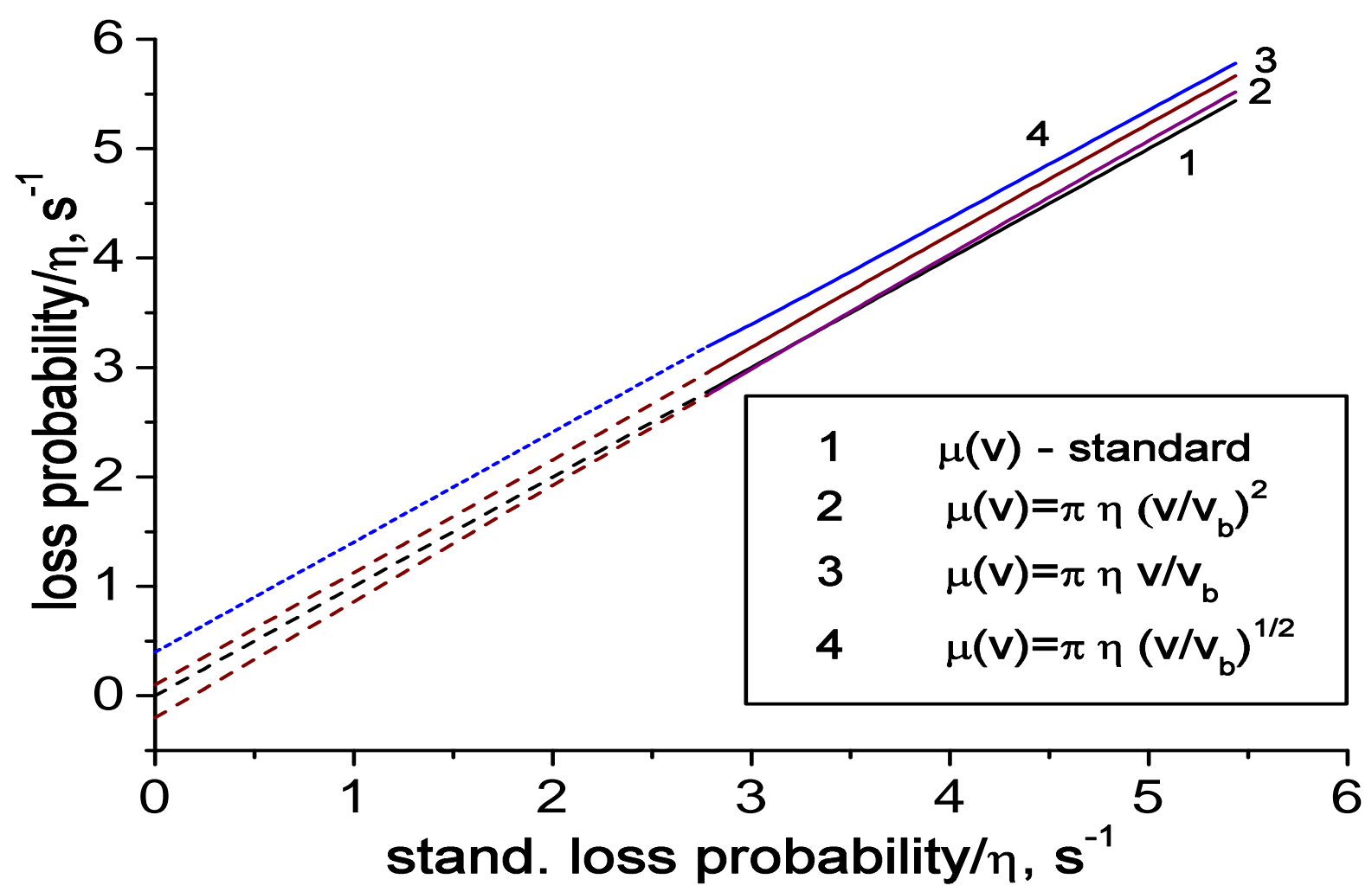

Figure 9: The same but for the Maxwellian tail spectrum and different UCN dependent UCN losses.

energy dependence of Eq. (2), of different energy dependent terms in the UCN loss, as a function of the calculated UCN loss for the standard, Eq. (2) formula.

The UCN spectrum was assumed to correspond to the tail of the Maxwell-like spectrum in these calculations. The extrapolated losses to zero energy differ from zero in the cases, when the neutron losses are not standard, by the value $\sim 10^{-6} s^{-1} \approx 10^{-3} \tau_{n}^{-1}$ if the $\eta=2 \times 10^{-6}$ in the worst case of the square root UCN energy dependence of the UCN loss coefficient.

It is obvious that for higher precision the loss corrections have to be evaluated more precisely and should be made as low as possible.

The low loss probability per one neutron collision with the wall is expected from the result of recent experiment [17], where it was obtained $\eta \approx 2 \times 10^{-6}$ for the low temperature fluorinated oil (PFPE), and there is hope to decrease further this value below $10^{-6}[27,28]$. On the other hand, larger size storage traps compared to [17] should decrease the loss rate due to a decrease of the wall collision rate.

\section{THE "ACCORDION" TRAP}

The effects of gravity on the storage of UCN in material traps with neutron losses have been considered first in [22] and in [29] with implications for experiments to determine the neutron lifetime. The 
consideration in [29] was focused on rectangular traps similar to the used in the experiments [12,16]. In these experiments the extrapolation to zero losses was performed by changing the length of the trap in horizontal direction.

An interesting proposal in the paper [29] was to use the UCN trap in the form of a bellows with horizontal axis. When the length of bellows and consequently the volume of the trap is changed, the surface is remained unchanged. In this case extrapolation to zero losses is straightforward. The project to realize this approach is published in [30].

The model calculations of the UCN losses in wall collisions as a function of the length of the bellows are presented below.

In the absence of gravity the accordion geometry is ideal in the sense of extrapolations to the infinite volume. In the presence of gravity the upper and lower parts of the trap are not equivalent, and the change of the form of the trap may, in principle, give some nonzero systematic effect for the value of extrapolated UCN losses.

The exact geometric form of the surface for different stretches of the bellows is not known. Here two models are used: the linear model (truncated cones) and the sinusoidal one. One segment for the linear model of the bellows is shown in Fig. 10.

It is assumed that at the stretching of the bellows the medium radius of the bellows is unchanged but the outer $R_{\max }$ and the inner $R_{\min }$ radii are changed according to the following expressions:

$$
\begin{array}{r}
d=H / N, \quad \sin \theta=d / l, \quad r=R_{0}+\frac{d}{\tan \theta}\left(\frac{x}{d}-\frac{1}{2}\right), \\
z=r \cos \varphi, \quad R_{\text {max }, \text { min }}=R_{0} \pm \frac{d}{2 \tan \theta},
\end{array}
$$

where $H$ is the length of the bellows, $N$ is the number of truncated conic segments of the bellows, $d$ is the length of the segment in horizontal direction, $R_{0}$ is the medium radius of the bellows, $l$ is the length of the generatrix of the conic segment, $\theta$ is the deformation angle, $\varphi$ is the angle around horizontal axis of the bellows. In this case the datum in $z$ direction is at the axis of the bellows.

Similar to the calculation for other UCN trap forms we have:

$$
\begin{array}{r}
S(y)=N \cdot S_{\mathrm{con}}(y)+2 \cdot S_{\mathrm{pl}}(y), \\
S_{\mathrm{con}}(y)=\frac{1}{\sin \theta} \int_{0}^{d} d x \int_{0}^{\pi} r \kappa\left(\sqrt{y^{2}-2 g r \cos \varphi / v_{b}^{2}}\right) d \varphi, \\
S_{\mathrm{pl}}(y)=\int_{-R_{0}}^{R_{0}} \kappa\left(\sqrt{y^{2}-2 g z / v_{b}^{2}}\right) \sqrt{2 R_{0} z-z^{2}} d z, \\
V(y)=2 N \int_{0}^{d} d x \int_{-r}^{r} \sqrt{y^{2}-2 g z / v_{b}^{2}} \sqrt{2 r z-z^{2}} d z,
\end{array}
$$

where $S_{\text {con }}(y)$ is the surface loss on one conic section of the bellows, $S_{\mathrm{pl}}(y)$ is the surface losses at the two disc surfaces of the bellows, $V(y)$ is the effective volume term. 


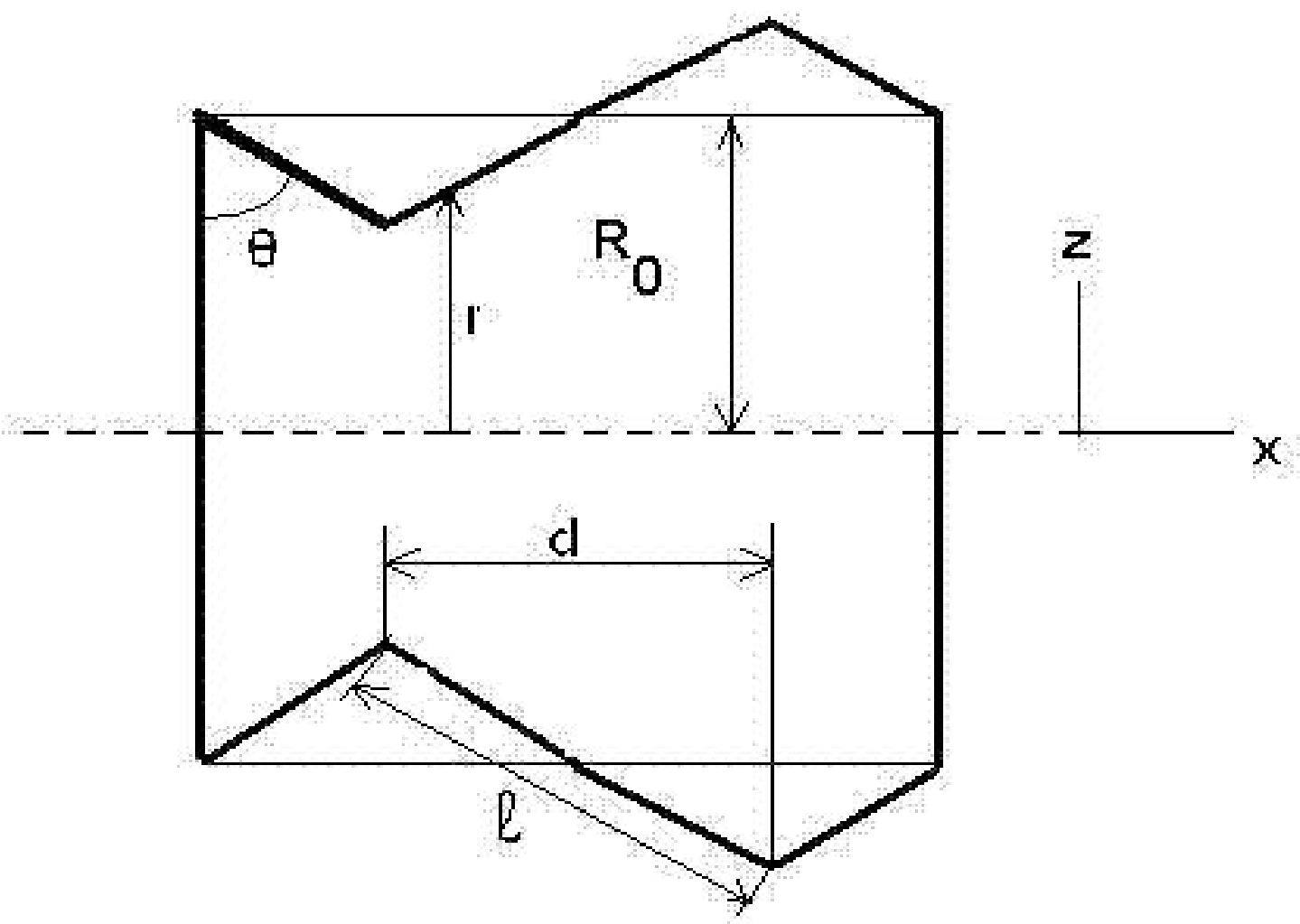

Figure 10: Schematic view of the "accordion" segment. 


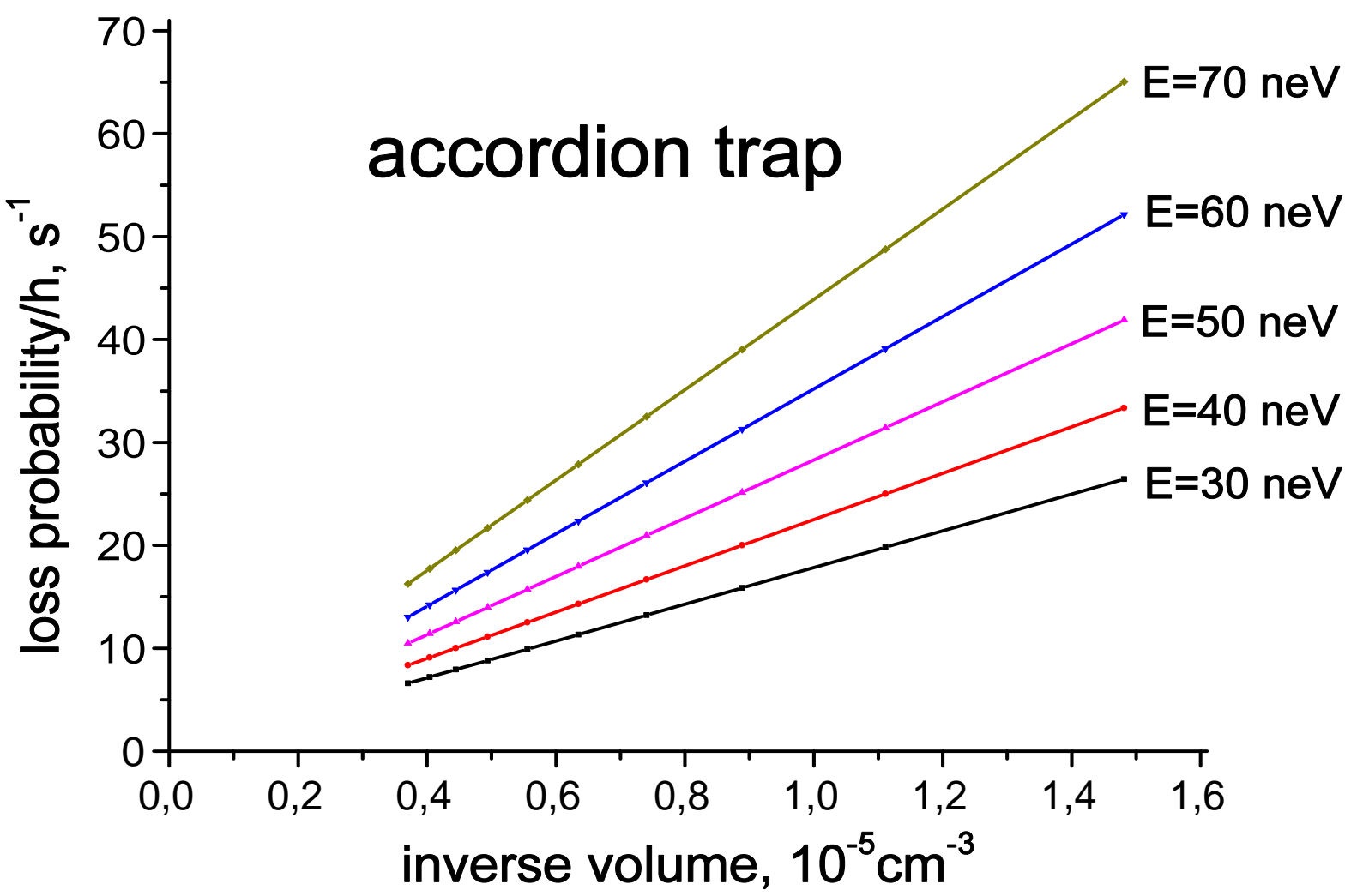

Figure 11: Reduced UCN loss probability in the "accordion" trap for different UCN energies as a function of the inverse volume of the trap. The datum is placed at the axis of the trap.

The results of calculations of the loss rate as a function of the inverse volume of the bellows is shown in Fig. 11. The parameters of the trap were taken from the Ref. [30]: $l=2.9 \mathrm{~cm}, R_{0}=$ $\left(R_{\max }+R_{\min }\right) / 2=26.75 \mathrm{~cm}$, the length was varied between 30 and $120 \mathrm{~cm}$.

Linear extrapolation $\gamma(1 / V)=\gamma_{\mathrm{inf}}+b \times(1 / V)$ for each of the energies gave $\gamma_{\mathrm{inf}} \approx(-5 \pm 5) \times 10^{-3}$. At the loss coefficient $\eta=10^{-5}$ the systematic effect arising from the deformation of the UCN storage volume at the size extrapolation is about $5 \times 10^{-8} \mathrm{~s}^{-1}$ for the inverse lifetime or $\approx 0.04 \mathrm{~s}$ for the neutron lifetime and does not depend on energy.

At larger value of $l$ the systematic effect at the extrapolation to infinite volume is increased.

The serious problem in the use of bellows UCN trap is deposition of the low temperature coverage at the corrugated surface and its stability at the deformation of the bellows. Another disadvantage consists in the narrow energy range of confined neutrons $\approx 30-70 \mathrm{neV}$ for the datum placed at the axis of the trap and in the case of the PFPE coverage for the bellows size of [30]. These limits follow from the conditions that the UCN should have the energy sufficient to reach the highest points of the trap and not exceed the boundary energy of the PFPE $E_{b} \approx 106 \mathrm{neV}$. 


\section{MAGNETIC TRAPS}

One of the most interesting methods of slow neutron storage has been proposed and experimentally demonstrated by Paul and collaborators [31,10]. It was a toroidal hexapole magnetic trap for very slow neutrons in the $\mu \mathrm{eV}$ energy range: the neutrons with longitudinal velocities between 8 and $15 \mathrm{~m} / \mathrm{s}$ and radial velocity spread up to $4 \mathrm{~m} / \mathrm{s}$ were stored in the ring with the diameter of $\sim 110 \mathrm{~cm}$. The neutron injection from the neutron guide into the magnetic ring was performed with the help of fast pneumatically driven neutron mirror reflector. The final experiments, in which the neutron lifetime was measured with the precision $10 \mathrm{~s}$, were performed at the very cold neutron channel of the PF-2 neutron source [32] of the ILL High Flux Reactor.

The great advantage may be achieved using pulsed injection of neutrons from the pulse neutron source [33,34]. In this method the neutron bunch from the UCN converter after spreading over the neutron guide can be trapped in the UCN cavity with high efficiency.

The detailed MCNP calculations for a solid para-hydrogen converter with an optimal cold orthohydrogen premoderator located in the radial channel of the MARK-2 TRIGA reactor yield the production of $\sim 10^{8}$ neutrons/Mole/10 MJ reactor pulse in the neutron velocity half-cylinder with the radius of 4 $\mathrm{m} / \mathrm{s}$ and the longitudinal velocity interval from 8 to $15 \mathrm{~m} / \mathrm{s}$. Possible size of one-mole para-hydrogen converter: the diameter of $7 \mathrm{~cm}$, the thickness of $0.5 \mathrm{~cm}$ is optimal for the reactor radial channel and for the neutron extraction in this energy range.

At the distance $L$ from the neutron source to the entrance to the storage ring, and the neutron velocity interval of stored neutrons between $v_{1}$ and $v_{2}$, the length of the neutron bunch passed this distance is $L\left(\left(v_{2} / v_{1}\right)-1\right)$. The length of the neutron guide at the TRIGA reactor from the moderator to the entrance of the magnetic ring may be as short as about $5-6 \mathrm{~m} / \mathrm{s}$, and at the length of the storage ring $\sim 350 \mathrm{~cm}$ [31] practically optimal condition may be realized for the neutron injection: almost all the bunch in the longitudinal velocity range $8-15 \mathrm{~m} / \mathrm{s}$ fills the storage ring during one reactor pulse. With larger storage ring and consequently larger orbit time the injection and storage will be more effective.

The neutron injection efficiency is not reported in [31]. In an assumption that the neutron survival during transport through the neutron guide and injection is only $1 \%$ it is expected up to $10^{6}$ neutrons trapped in the ring per one filling. It is three orders of the magnitude larger than reported in [31].

The additional advantage consists in the possibility to variate the spectrum of injected neutrons with help of the pulse injector due to time-of-flight separation of neutrons in the neutron guide.

Similar possibility of pulsed neutron filling from pulse neutron source exists for other types of the magnetic traps for ultracold neutrons [35] if one provides the magnetic trap with pulse magnetic shutter.

It seems possible, in principle, to control neutron losses from magnetic ring by placing very slow neutron detector based on the low temperature scintillator (for example pure CsI [36]) at the outer side of the ring storage volume and PMT in the central part of the ring.

Another possibility - the decay proton counting is possible placing proton counters in the center of 
the storage ring and introducing accelerating electric field between the walls of the storage volume and the proton detectors.

The author is grateful to Dr. V. K. Ignatovich for clarifications concerning his work [22].

References

1. H. Abele, Prog. Part. Nucl. Phys. 60, 1 (2008).

2. N. Severijns, M. Beck and O. Naviliat-Cuncic, Rev. Mod. Phys. 78, 991 (2006).

3. J. S. Nico and W. M. Snow, Ann. Rev. Nucl. Part. Sci. 55, 27 (2005); nucl-ex/0612022.

4. L. N. Bondarenko, V. V. Kurguzov, Yu. A. Prokof'ev, et al., Pis'ma v ZhETF 28, 329 (1978) [JETP Lett. 28, 303 (1978)]; P. E. Spivak, ZhETF 94, 1 (1988) [JETP 67, 1735 (1988)].

5. J. Byrne, P. G. Dawber, J. A. Spain, et al., Phys. Rev. Lett. 65, 289 (1990).

6. J. Byrne, P. G. Dawber, C. G. Habeck, et al., Europhys. Lett. 33, 187 (1996).

7. M. S. Dewey, D. M. Gillian, J. S. Nico, et al., Phys. Rev. Lett. 91, 152302 (2003).

8. J. S. Nico, M. S. Dewey, D. M. Gillian, et al., nucl-ex/0411041

9. M. S. Dewey, D. M. Gillian, J. S. Nico, et al., Phys. Rev. C 71, 055502 (2005).

10. F. Anton, W. Paul, W. Mampe, et al., Z. Phys. C 45, 25 (1989).

11. A. G. Kharitonov, V. V. Nesvizhevsky, A. P. Serebrov, et al., Nucl. Instrum. Methods A 284, 98 (1989).

12. W. Mampe, P. Ageron, J. C. Bates, et al., Nucl. Instrum. Methods A 284, 111 (1989); Phys. Rev. Lett. 63, 593 (1989).

13. V. V. Nesvizhevsky, A. P. Serebrov, R. R. Tal'daev, et al., ZhETF 102, 740 (1992); [JETP 75, 405 (1992)].

14. W. Mampe, L. N. Bondarenko, V. I. Morozov, et al., Pis'ma v ZhETF 57, 77 (1993); [JETP Lett. 57, 82 (1993)].

15. S. Arzumanov, L. Bondarenko, S. Chernyavsky, et al., Phys. Lett. B 483, 15 (2000).

16. A. Pichlmaier, J. Butterworth, P. Geltenbort, et al., Nucl. Instrum. Methods A 440, 517 (2000); A. Pichlmaier, Dissertation (TU München, 1999).

17. A. Serebrov, V. Varlamov, A. Kharitonov, et al., Phys. Lett. B 605, 72 (2005); nucl-ex/0408009; A. P. Serebrov, V. E. Varlamov, A. G. Kharitonov, et al. Phys. Rev. C 78, 035505 (2008).

18. V. Ezhov, in The Proceeedings of the VI International Conference "Ultracold and Cold Neutrons, Physics and Sources" (St. Petersburg, Moscow, 2007), http://cns.spb.ru/6UCN/proceed.html.

19. V. Ezhov, in The Proceeedings of the VII International Conference "Ultracold and Cold Neutrons, Physics and Sources” (St. Petersburg, 2009), http://cns.spb.ru/7UCN/proceed.html.

20. Rev. Part. Phys., Phys. Lett. B 667, 1070 (2008).

21. Yu. Yu. Kosvintsev, Yu. A. Kushnir, V. I. Morozov, et al., Pis'ma v ZhETF 31, 257 (1980) [JETP Lett. 31, 236 (1980)]. 
Yu. Yu. Kosvintsev, V. I. Morozov, G. I. Terekhov, Pis'ma v ZhETF 36, 346 (1982) [JETP Lett. 36, $424(1982)]$.

Yu. Yu. Kosvintsev, V. I. Morozov, G. I. Terekhov, Pis'ma v ZhETF 44, 444 (1986) [JETP Lett. 44, 571 (1986)].

22. V. K. Ignatovich, G. I. Terekhov, JINR Commun. P4-9567 (Dubna, 1976).

23. L. V. Groshev, V. N. Dvoretsky, A. M. Demidov, et al., JINR Preprint P3-9534 (Dubna, 1976).

24. D. J. Richardson, J. M. Pendlebury, P. Iaydjiev, et al., Nucl. Instrum. Methods A 308, 568 (1991).

25. A. Serebrov, A. Fomin, I. Shoka, et al., "New project of neutron lifetime measurement with gravitational trap of UCN.", in The Proceedings of the VI-th International Conference "UltraCold and Cold Neutrons, Physics and Sources”, (St. Petersburg, Moscow, 2007), http://cns.spb.ru/6UCN/proceed.html.

26. Yu. N. Pokotilovski, ZhETF 123, 203 (2003) [JETP 96172 (2003)].

27. Yu. N. Pokotilovski, Nucl. Instrum. Methods A 554, 356 (2005).

28. Yu. N. Pokotilovski, I. Natkaniec and K. Holderna-Natkaniec, Physica B 403, 1942 (2008).

29. J. M. Pendlebury and D. J. Richardson, Nucl. Instrum. Methods A 337, 504 (1994).

30. B. Yerozolimsky, A. Steyerl, O. Kwon et al., J. Res. Natl. Inst. Stand. Technol. 110, 351 (2005); and in The Proc. XII Intern. Seminar on Interaction of Neutrons with Nuclei (Dubna, May 26-29, 2004), p.222.

31. K.-J. Kügler, W. Paul, and U. Trinks, Phys. Lett. 72B, 422 (1978).

K.-J. Kügler, K. Moritz, W. Paul et al., Nucl. Instrum. Methods 228, 240 (1978).

32. A. Steyerl, H. Nagel, F.-X. Schreiber, et al., Phys. Lett. A 116, 347 (1986).

33. Yu. N. Pokotilovski, Nucl. Instrum. Methods A 356, 412 (1995).

34 A. Frei, Y. Sobolev, I. Altarev, et al., Eur. Phys. Journ. A 34, 119 (2007).

35. V. V. Vladimirskii, ZhETF 39, 1062 (1960) [Sov. Phys. JETP 12, 740 (1960)].

Yu. G. Abov, S. P. Borovlev, V. V. Vasil'ev, et al., Yad. Fiz. 38, 122 (1983) [Sov. J. Nucl. Phys. 38, 70 (1983)].

V. F. Ezhov, A. Z. Andreev, A. A. Glushkov et al., Journ. Res. NIST 110, 345 (2005).

R. Picker, I. Altarev, J. Bröker, et al., J. Res. NIST 110, 357 (2005).

J. D. Bowman and S. I. Pentila, J. Res. NIST 110, 361 (2005).

36. J. B. Birks, The Theory and Practice of Scintillation Counting (Pergamon Press, Oxford, 1964), p. 460. 